\title{
Iteration Procedures for the Dirichlet Difference Problem
}

Introduction. A fundamental paper in the theory of solving partial differential equations by iteration has been written by FrankEL. ${ }^{1}$ In this paper Frankel discusses the RICHARDSON and LIEBMANN procedures and their corresponding accelerated procedures. The latter are termed the extrapolated Liebmann and the second-order Richardson procedures. From his paper three disadvantages of the second-order Richardson procedure as compared to the extrapolated Liebmann procedure are implied.

(1) The extrapolated Liebmann procedure appears to converge twice as fast.

(2) The extrapolated Liebmann procedure seems to require fewer operations for each step.

(3) The second-order Richardson procedure appears to require the memory of two sets of values as opposed to only one set for the extrapolated Liebmann procedure.

In the present paper all of these disadvantages will be removed for the case of the Laplace difference equation. The disadvantage of the simple Richardson procedure versus the simple Liebmann procedure will also be removed. Since the Richardson procedures do not require rapid access memory and also have simpler error eigenfunctions, the Liebmann procedures will no longer be viewed as superior (see, e.g. SHELdon \& Thомas ${ }^{5}$ ).

In addition it will be shown that knowledge of the optimum value of the parameter(s) for either accelerated procedure immediately determines the optimum value of the parameter(s) for the other accelerated procedure. These parameters will be determined if the maximum eigenvalue of the simple Richardson procedure is known, and some means based on this for estimating these parameters for regions other than rectangles will be presented. These regions will be bounded by vertical or horizontal lines connecting the equally-spaced lattice points. Curvilinear boundaries may be approximated by such boundaries.

The notation of FRANKEL ${ }^{1}$ will be used and some familiarity with this paper will be assumed.

Some ideas presented here will be of small use if an entirely electronic computer is being used, as they will be expected to save only a few iterations. However, they will be of interest if a punched card machine is being used.

The Richardson Method. In this method the correction process applied to each $\phi^{n}$ consists of the addition of a positive multiple of $L \phi^{n}$ (for each interior point). Thus

$$
\begin{aligned}
\phi_{j, k}^{n+1} & =\phi_{j, k}^{n}+\alpha L \phi_{j, k}^{n} \\
& \equiv \phi_{j, k}^{n}+\alpha\left[\phi_{j-1, k}^{n}+\phi_{j+1, k}^{n}+\phi_{j, k-1}^{n}+\phi_{j, k+1}^{n}-4 \phi_{j, k}^{n}\right]
\end{aligned}
$$


The error at each stage is

$$
\epsilon_{j, k}^{n} \equiv \phi_{j, k}^{n}-\phi_{j, k} .
$$

The error eigenfunctions of the operator $L$ for a $p \times q$ rectangle are:

$$
\epsilon_{j, k}^{(r, s)}=\sin (\pi r j / p) \sin (\pi s k / q) \quad\left\{\begin{array}{l}
r=1,2, \cdots, p-1 \\
s=1,2, \cdots, q-1
\end{array}\right.
$$

The corresponding eigenvalues are

$$
L_{(r, 8)}=-4\left[\sin ^{2}(\pi r / 2 p)+\sin ^{2}(\pi s / 2 q)\right] .
$$

The eigenvalues $L_{(r, s)}$ of the Laplacian operator $L$ and the function $t \equiv \cos (\pi r / p)+\cos (\pi s / q)$, which is defined in the second of equations (27) of Frankel, ${ }^{1}$ satisfy the relation,

$$
L_{(r, s)} \equiv-4+2 t .
$$

Therefore the eigenvalues of the iteration operator $K \equiv 1+\alpha L$ are

$$
K_{(r, s)} \equiv 1-4 \alpha+2 \alpha t .
$$

In particular, for $\alpha=\frac{1}{4}$ (shown in Frankel ${ }^{1}$ to be the optimum $\alpha$ ),

$$
K_{(r, s)}=t / 2 \text {. }
$$

For convenience denote the eigenvalues of the simple Richardson operator with $\alpha=\frac{1}{4}$, for any region, by $t / 2$. It will be seen that these eigenvalues are intimately connected with the eigenvalues of the other iteration processes and since they are the simplest they will be referred to as the "reference" eigenvalues.

If $\epsilon_{j, k}$ is an error eigenfunction of the simple Richardson process (formula (5) of Frankel ${ }^{1}$, with eigenvalue $t / 2$, then $(-1)^{i+k} \epsilon_{j, k}$ is also an error eigenfunction with eigenvalue $-t / 2$. Thus the non-zero reference eigenvalues occur in pairs with the same absolute value but opposite sign.

It follows that if $L_{0}$ and $L_{m}$ are the smallest and largest in magnitude of the $L_{(r, s)}$, then

or

$$
\frac{1}{2} t_{\max }=1+\frac{1}{4} L_{0}=-\left[1+\frac{1}{4} L_{m}\right]=-\left(-\frac{1}{2} t_{\max }\right)
$$

$$
L_{0}+L_{m} \equiv-8 .
$$

See also MiLne ${ }^{8}$, section 85.

In Frankel ${ }^{1}$ it is shown that for $\alpha=\frac{1}{4}$ (the optimum value) the error eigenfunctions of lowest and highest frequency decrease at the slowest rate. But there is an $\alpha$ which removes the latter completely, still decreasing all components. This $\alpha$ is

$$
\alpha_{0}=1 /\left(4+2 t_{\max }\right) .
$$

Even if $t_{\max }$ is unknown, an $\alpha$ slightly larger than $\frac{1}{8}$ is sure to strike a heavy blow at this highest frequency error, as well as at other high frequency errors. For example, for a $20 \times 20$ square, $\alpha_{0}$ is, to five places, 0.12577 . For every eigenfunction corresponding to a $t \leq 0$, there is an $\alpha$ in the range $\frac{1}{8}<\alpha \leq \frac{1}{4}$ such that this removes completely that eigenfunction. [Of course, there is an $\alpha$ which will remove any specific eigenfunction, but unfortunately when $t \gg 0$ the $\alpha$ is such that the higher frequency errors (always present 
due to round off) may increase tremendously.] If the problem is first solved using a coarse grid and then values assigned by interpolation, the error components will tend to be of the higher frequency type, and may be reduced in this manner. (See Flanders \& ShorTLEY ${ }^{2}$, p. 1330.)

In this paper ${ }^{2}$ the lowest frequency eigenfunction has been picturesquely termed a "pillow function." This component of the error is the most troublesome to the iteration process, and the initial values should guard against it as much as possible. Thus assigning the average of the boundary values for all initial values will in general be better than assigning all zeros. It can be shown that there always exists a constant value for $\phi_{j, \boldsymbol{k}}^{0}$ which has no pillow function error, and the average of the boundary values is a reasonable approximation to this constant. This step corresponds to a block relaxation of Southwell. ${ }^{6,7}$

Further block relaxations may be used (especially if the region has lines of symmetry) to reduce initially other low frequency error components.

The nearness of $\frac{1}{2} t_{\max }$ to unity for regions with a large number of interior points is an indication of the condition (see TAUSSKY ${ }^{10}$ ) of the system of linear equations. If the remaining error is of low frequency, it may seem that the difference equations are satisfied even though the approximate solution is far from the true solution. A more sensitive test is the following. It is an analogue ${ }^{12}$ of the theorem on the vanishing of the integral of the normal derivative of a harmonic function around a closed path, and is obtained by summing the entire set of difference equations for the region. The result is that the sum of the values at the boundary points must equal the sum of the values at the interior points adjacent to the boundary, with the exception that at corners some values are counted twice and some not at all, in an obvious manner. The points are merely paired at opposite ends of segments of grid lines, and those having no mate are omitted. This principle must also be satisfied for all paths interior to the region, and may be used as a check for any low frequency error.

Even if one desires to use the other methods described in Frankel, ${ }^{1}$ the usefulness of the simple Richardson method is not at an end. The optimum $\alpha$ and the optimum $\alpha$ and $\beta$ in these methods will be shown to be determined by the reference eigenvalue of the pillow function. This eigenvalue is $\frac{1}{2} t_{\max }$.

FLANDERS \& SHORTLEY ${ }^{2}$ give a method for determining this eigenvalue experimentally. One assumes zero boundary values and approximates a pillow function. By iteration or by the non-iteration method of this paper one attempts to remove the other components present and then the rate at which the pillow function is reducing is its eigenvalue. A good approximation to the eigenvalue can then be obtained by iterating once more with $\alpha=\frac{1}{4}$ and using the Rayleigh quotient formula:

$$
\frac{t_{\max }}{2} \cong \frac{\sum_{j, k} \phi_{j, k}^{n+1} \phi_{j, k}^{n}}{\sum_{j, k}\left(\phi_{j, k}^{n}\right)^{2}} \quad \text { or } \quad \frac{t_{\max }}{2} \cong \frac{\sum_{j, k}\left(\phi_{j, k}^{n+1}\right)^{2}}{\sum_{k} \phi_{j, k}^{n+1} \phi_{j, k}^{n}} .
$$

A check on the results is presented here. If one actually has the pillow function, multiplying it by $(-1)^{i+k}$ (changing the sign at alternate points) will produce the eigenfunction of highest frequency, and then $\alpha_{0}$ (formula $(D)$ ) will reduce it to zero in one iteration. 
If one desires to solve a complete problem by the simple Richardson process, it may be observed that two independent computations are being carried on when using the optimum $\alpha$ of $\frac{1}{4}$, either of which may be omitted. This effectively doubles the convergence rate. The formula is

$$
\phi_{j, k}^{n+1}=\frac{1}{4}\left[\phi_{j-1, k}^{n}+\phi_{j+1, k}^{n}+\phi_{j, k-1}^{n}+\phi_{j, k+1}^{n}\right] .
$$

Since $\phi_{j, k}^{n}$ is not used, it need not be computed. This means that $\phi_{j, k}^{0}$ may be assigned at points where $j$ and $k$ have, say, like parity (both even or both odd). Then $\phi_{j, k}^{1}$ is computed at the points where $j$ and $k$ have unlike parity. In general, $\phi_{j, k}^{n}$ is computed for even $n$ at points where $j$ and $k$ have like parity, and for odd $n$ at points where $j$ and $k$ have unlike parity. The method then becomes a Liebmann method in the sense that latest values are always used.

The Liebmann Method $\left(\alpha=\frac{1}{4}\right)$. In the Liebmann method the new value of $\phi_{j, k}^{n+1}$ is used as soon as it is computed. Thus

$$
\phi_{j, k}^{n+1}=\phi_{j, k}^{n}+\alpha\left[\phi_{j-1, k}^{n+1}+\phi_{j+1, k}^{n}+\phi_{j, k-1}^{n+1}+\phi_{j, k+1}^{n}-4 \phi_{j, k}^{n}\right] .
$$

With $\alpha=\frac{1}{4}$, this becomes

$$
\phi_{j, k}^{n+1}=\frac{1}{4}\left[\phi_{j-1, k}^{n+1}+\phi_{j+1, k}^{n}+\phi_{j, k-1}^{n+1}+\phi_{j, k+1}^{n}\right] .
$$

The Extrapolated Liebmann Method. In Frankel ${ }^{1}$ it is shown that the optimum $\alpha$ (equation above) is determined by the equation (35)

$$
\alpha^{2} t_{\max }^{2}-4 \alpha+1=0 .
$$

For the rectangle, $t_{\max }=\cos (\pi / p)+\cos (\pi / q)$. But for other regions, $t_{\max }$ is not in general known, except that it is twice the reference eigenvalue of the pillow error eigenfunction. This has not been proved here, but it follows as a corollary of results presented in Young. ${ }^{3}$ It is shown there that it is better to overestimate the optimum $\alpha$ than to underestimate it. Further discussion of the problem of obtaining the optimum $\alpha$ will be presented in a later section.

The Second-Order Richardson Method. The iteration equation for the second-order Richardson method is:

$$
\phi_{j, k}^{n+1}=\phi_{j, k}^{n}+\alpha L \phi_{j, k}^{n}+\beta\left(\phi_{j, k}^{n}-\phi_{j, k}^{n-1}\right) .
$$

It appears that two lines of starting values are necessary. It is not clear what relation they must bear to one another or how this relation will affect later iterations. [If the coefficient of an error eigenfunction is $A$ in $\phi_{j, \mathbf{k}}^{n-1}$ and $a$ in $\phi_{j, k}^{n}$, its coefficient in $\phi_{j, k}^{n+1}$ will be $a(1-4 \alpha+\beta+2 \alpha t)-\beta A$. For $\beta=4 \alpha-1$ this reduces to $2 \alpha a t+(1-4 \alpha) A]$. Actually this method assumes that the simple Richardson method has the sequence of values "going in the right direction" and therefore pushes them a little further.

Formula (45) of FRANKEL ${ }^{1}$ has a misprint. It should read

$$
K^{*} \cong 1-\pi\left(p^{-2}+q^{-2}\right)^{\frac{1}{2}} / \sqrt{2} .
$$

In the third line below this formula the word "advantage" should be "disadvantage." This disadvantage, number (1) of the introduction, will now be removed. Also the above initial difficulty will disappear. 
One of the formulas defining the optimum $\alpha$ and $\beta$ in Frankel ${ }^{1}$ is

$$
\alpha\left(L_{0}+L_{m}\right)=-2(1+\beta) .
$$

By formula (C) this simplifies to

$$
\beta=4 \alpha-1 .
$$

The other formula in Frankel ${ }^{1}$ is

or

$$
\alpha L_{0}=-\left(1-\beta^{\mathbf{k}}\right)^{2}
$$

By formula (A)

$$
1+\alpha L_{0}+\beta=2 \beta^{\frac{1}{1}} \text {. }
$$

Thus

$$
L_{0}=-4-2 t_{\max } \text {. }
$$

or

$$
1-4 \alpha-2 \alpha t_{\max }+4 \alpha-1=2(4 \alpha-1)^{\frac{1}{2}}
$$

But this is the same equation as equation (35) of Frankel. ${ }^{1}$ Thus the optimum $\alpha$ in a given region is the same in the second-order Richardson method as in the extrapolated Liebmann method.

Note that as $t_{\max } \rightarrow 2$, the optimum $\alpha \rightarrow \frac{1}{2}$.

Substituting $4 \alpha-1$ for $\beta$ and writing out the operator $L$ in full, the iteration process becomes:

$$
\begin{aligned}
\phi_{j, k}^{n+1}=\phi_{j, k}^{n}+\alpha\left[\phi_{j-1, k}^{n}+\phi_{j+1, k}^{n}+\phi_{j, k-1}^{n}+\phi_{j, k+1}^{n}\right. & \left.-4 \phi_{j, k}^{n}\right] \\
& +(4 \alpha-1)\left(\phi_{j, k}^{n}-\phi_{j, k}^{n-1}\right)
\end{aligned}
$$

or

$$
\phi_{j, k}^{n+1}=\alpha\left[\phi_{j-1, k}^{n}+\phi_{j+1, k}^{n}+\phi_{j, k-1}^{n}+\phi_{j, k+1}^{n}\right]+(1-4 \alpha) \phi_{j, k}^{n-1} .
$$

The formula has already simplified considerably. Disadvantage (2) of the introduction has disappeared. $\phi_{j, k}^{n}$ has dropped out. As in the simple Richardson method, it is unnecessary to compute it at all. That is, if one has values at alternate points, say where $j+k$ is odd, and values in the next iteration at points where $j+k$ is even, then one can compute the values in the succeeding iteration at points where $j+k$ is odd, and from these the values in the iteration after that at points where $j+k$ is even again. The missing values are independent of the values computed, and vice versa. Thus only half the computation is necessary. This cancels the factor of two disadvantage over the extrapolated Liebmann method.

This method of computing removes another disadvantage, number (3) of the introduction, of the second-order Richardson method for machines with limited memory. At no time is it necessary to remember more than one value at any point. The method is a Liebmann method in the sense that latest values are always used. In fact this method and the extrapolated Liebmann method are both special cases of the "successive overrelaxation method" developed by Young, ${ }^{3}$ depending only on "ordering" of the points. This reduced second-order Richardson method is actually produced by an "alternate diagonal" ordering of Young. ${ }^{3}$ However, there is one difference, 
which can perhaps be used to advantage. In one case there are "missing" values. In the other case two successive iterations are collapsed into one. This of course changes the eigenfunctions. In the reduced second-order Richardson method, the eigenfunctions are still the simple Richardson eigenfunctions.

Use of a Richardson procedure now permits the lowest frequency error components to be attacked both by initial block relaxations and by solution first with a coarse grid. The highest frequency error component can be eliminated in one iteration by the simple Richardson procedure with $\alpha=\alpha_{0}$ as given by formula (D) and other high frequency components can be effectively eliminated in a like manner.

However, unlike the simple Richardson procedure, for the second order Richardson procedure all error components become equally "troublesome," at least in the simple sense of asymptotic decay rate. ${ }^{11}$ Attacking them individually no longer gives such an obvious advantage. However, examine the expression $2 \alpha a t+(1-4 \alpha) A$ (given above in brackets) for the coefficient of an error eigenfunction in terms of its coefficients in the two previous lines. Observe that one will probably obtain a second line from an initial approximation by an iteration using the simple Richardson procedure with $\alpha=\frac{1}{4}$. Then $a=A t / 2$ and the expression becomes $\left(\alpha t^{2}+1-4 \alpha\right) A$. Considered as a function of $t$ this is a parabola with minimum at $t=0$. This suggests that one will probably gain by making some effort to eliminate the lowest and highest frequency components and then iterating at least twice by the simple Richardson procedure with $\alpha=\frac{1}{4}$ before starting the second order procedure (assuming, of course, that the computer being used is not of the highest speed).

The Reference Eigenvalue for the Pillow Function. Since the reference eigenvalue for the pillow function is $\frac{1}{2} t_{\max }$ and the optimum $\alpha$ in the faster converging methods is determined by the smaller root of the equation

$$
\alpha^{2} t_{\max }^{2}-4 \alpha+1=0,
$$

this eigenvalue if of prime importance. A practical method for approximating its value, using the Rayleigh quotient, has already been mentioned. Unfortunately this method in general gives a value which is too small, and as mentioned previously, it is much better to have a value which is too large. Since a formula is known for the rectangle, it is useful to observe that if the region in question is covered by a rectangle, the desired value is less than the reference pillow eigenvalue of the rectangle. Similarly a rectangle interior to the region will have a smaller reference pillow eigenvalue. In making these estimates, better results can be obtained if it is realized that for a fixed number $M$ of interior points the reference pillow eigenvalue is a measure of the compactness of the region. E.g., an unbounded number of interior points can be strung out so that the maximum reference eigenvalue is less than one-half. But only four interior points arranged in a square will have a reference eigenvalue of one-half. This means that any arms jutting out from the region can be cut off and used to fill in any part of the covering rectangle without reducing the maximum reference eigenvalue of the region in question. This also means that, unless the region is almost circular, an upper bound to the reference eigenvalue is $\cos \pi /\left(1+M^{\frac{1}{2}}\right)$, being the 
maximum reference eigenvalue of a square with $M$ interior points, and a square being a rather compact region.

Time Estimates. The tables of Frankel, ${ }^{1}$ page 74, can now be changed to:

\begin{tabular}{ll}
\multicolumn{1}{c}{ Method } & $T$ \\
Laplace-Richardson $\left(\alpha=\frac{1}{4}\right)$ & $4 \tau$ \\
Laplace-Liebmann $\left(\alpha=\frac{1}{4}\right)$ & $4 \tau$ \\
Laplace-Liebmann (optimum $\alpha)$ & $6 \tau$ \\
Laplace-2nd-order Richardson & $6 \tau$.
\end{tabular}

\section{Approximate Total Calculating Time.}

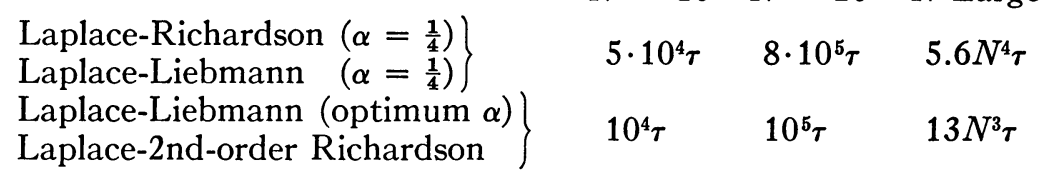

Here $\tau$ is the mean time required for each arithmetic operation, $T$ is the time for the evaluation of one $\phi_{j, \mathrm{k}}^{n}$ and $N$ is the common value of $p$ and $q$ for a square with $(N-1)^{2}$ interior points.

The change in the extrapolated Liebmann method (for optimum $\alpha$ ) from $7 \tau$ to $6 \tau$ in the first table is because terms may be collected in the iteration formula to give:

$$
\phi_{j, k}^{n+1}=\alpha\left[\phi_{j-1, k}^{n+1}+\phi_{j+1, k}^{n}+\phi_{j, k-1}^{n+1}+\phi_{j, k+1}^{n}\right]+(1-4 \alpha) \phi_{j, k}^{n}
$$

and $1-4 \alpha$ may be computed when $\alpha$ is computed.

The improved second-order Richardson method now has the same rate of convergence as the extrapolated Liebmann method, requires no more memory, and does not require rapid access memory. Thus it would seem to be a superior method for general use.

\section{JAMES D. RiLEY}

U. S. Naval Ordnance Lab.

Silver Spring, Md.

${ }^{1}$ Stanley P. Frankel, "Convergence rates of iterative treatments of partial differential equations," $M T A C$, v. 4, 1950, p. 65-75.

2 D. Flanders \& G. Shortley, "Numerical determination of fundamental modes." Jn. Appl. Phys., v. 21, 1950, p. 1326-1332.

${ }^{3}$ DAvID Young, "Iterative methods for solving partial difference equations of elliptic type," Amer. Math. Soc., Trans., v. 76, 1954, p. 92-111.

4 R. PLUNKETT, "On the rate of convergence of relaxation methods," Quart. of Appl. Math., v. 10, 1952, p. 263-266.

"John Sheldon \& L. H. Thomas, "The use of large scale computing in physics," Jn. Appl. Phys., v. 24, 1950, p. 235-242.

6 L.Fox, "A short account of relaxation methods," Quart. Jour. Mech. and Appl. Math., v. 1,1950 , p. $253-280$.

7 R. V. Southwell, Relaxation Methods in Theoretical Physics. Oxford, 1946.

${ }^{8}$ W. E. Milne, Numerical Solution of Differential Equations. New York, 1953.

9 G. SHORTLEY \& R. Weller, "The numerical solution of Laplace's equation," $J n$. Appl. Phys., v. 9, 1938, p. 334-348.

${ }^{10}$ Olga Taussky, "Note on the condition of matrices," MTAC, v. 4, 1950, p. 111-112.

11 The author is indebted to the referee at this point.

${ }^{12}$ FRANZ WoLF, "Über die angenäherte numerische Berechnung harmonischer und biharmonischer Funktionen," Zeit. angew. Math. Mech., v. 6, 1926, p. 118-150. 